\title{
Analyse désagrégée des facteurs environnementaux associés au nombre d'enfants blessés par un véhicule à moteur en milieu urbain
}

\section{A disaggregated analysis of environmental factors associated with the number of children injured by motor vehicles in urban settings \\ Análisis desagregado de los factores ambientales asociados al número de niños heridos por un vehículo motorizado, en medio urbano}

\author{
Patrick Morency, Lise Gauvin, François Tessier, Luis Miranda-Moreno, \\ Marie-Soleil Cloutier et Catherine Morency
}

Volume 55, numéro 156, décembre 2011

URI : https://id.erudit.org/iderudit/1008888ar

DOI : https://doi.org/10.7202/1008888ar

Aller au sommaire du numéro

Éditeur(s)

Département de géographie de l’Université Laval

ISSN

0007-9766 (imprimé)

1708-8968 (numérique)

Découvrir la revue

Citer cet article

Morency, P., Gauvin, L., Tessier, F., Miranda-Moreno, L., Cloutier, M.-S. \& Morency, C. (2011). Analyse désagrégée des facteurs environnementaux associés au nombre d'enfants blessés par un véhicule à moteur en milieu urbain. Cahiers de géographie du Québec, 55(156), 449-468.

https://doi.org/10.7202/1008888ar

\section{Résumé de l'article}

Cette recherche vise à décrire le nombre et la distribution géographique des enfants blessés par un véhicule à moteur en milieu urbain, ainsi que l'association avec des facteurs environnementaux reconnus. Elle porte sur 6775 enfants (5 à 17 ans) blessés sur le réseau routier montréalais de 1999 à 2008. Les analyses descriptives démontrent que, dans les arrondissements et municipalités les plus pauvres comme dans les plus riches, environ la moitié des enfants blessés aux intersections l'ont été à des sites ayant un volume de circulation très élevé ou sur une route majeure (artère). Le nombre moyen de jeunes piétons blessés est 39 fois plus élevé aux intersections ayant de forts volumes de circulation automobile ( $5^{\mathrm{e}}$ quintile) qu'à celles situées dans le $1^{\mathrm{er}}$ quintile. Des régressions locales pondérées illustrent la variation de l'effet du volume de circulation dans trois arrondissements centraux ayant une densité d'enfants élevée et relativement similaire. 


\section{Analyse désagrégée des facteurs environnementaux associés au nombre d'enfants blessés par un véhicule à moteur en milieu urbain}

\author{
A disaggregated analysis of environmental \\ factors associated with the number of \\ children injured by motor vehicles in urban \\ settings
}

Análisis desagregado de los factores ambientales asociados al número de niños heridos por un vehículo motorizado, en medio urbano

\author{
Patrick MORENCY \\ Direction de santé publique de l'Agence de la \\ santé et des services sociaux de Montréal \\ Pmorency@santepub-mtl.qc.ca \\ Lise GAUVIN \\ Département de médecine sociale et préven- \\ tive, Université de Montréal \\ Lise.Gauvin2@umontreal.ca \\ François TESSIER \\ Direction de santé publique de l'Agence de la \\ santé et des services sociaux de Montréal \\ Ftessier@santepub-mtl.qc.ca \\ Luis MIRANDA-MORENO \\ Department of Civil Engineering and Applied \\ Mechanics, McGill University \\ Luis.Miranda-Moreno@mcgill.ca \\ Marie-Soleil CLOUTIER \\ Centre Urbanisation, Culture et Société-INRS \\ Marie-Soleil.Cloutier@ucs.inrs.ca \\ Catherine MORENCY \\ Département des génies civil, géologique et \\ des mines, École Polytechnique de Montréal \\ Cmorency@polymtl.ca
}

\section{Résumé}

Cette recherche vise à décrire le nombre et la distribution géographique des enfants blessés par un véhicule à moteur en milieu urbain, ainsi que l'association avec des facteurs environnementaux reconnus. Elle porte sur 6775 enfants ( 5 à 17 ans) blessés sur le réseau routier montréalais de 1999 à 2008. Les analyses descriptives démontrent que, dans les arrondissements et municipalités les plus pauvres comme dans les plus riches, environ la moitié des enfants blessés aux intersections l'ont été à des sites ayant un volume de circulation très élevé ou sur une route majeure (artère). Le nombre moyen de jeunes piétons blessés est 39 fois plus élevé aux intersections ayant de forts volumes de circulation automobile ( $5^{\mathrm{e}}$ quintile) qu'à celles situées dans le $1^{\text {er }}$ quintile. Des régressions locales pondérées illustrent la variation de l'effet du volume de circulation dans trois arrondissements centraux ayant une densité d'enfants élevée et relativement similaire.

\section{Mots clés}

Blessures, piétons, cyclistes, enfants, sécurité routière, santé publique. 


\begin{abstract}
This research analyzes the number and geographic distribution of children injured by motor vehicles in urban settings as well as the association with recognized environmental factors such as traffic volume and street geometry. The sample includes 6,775 children (5 to 17 years old) injured on the Montreal road network between 1999 and 2008. Results show that, in both the poorest and the wealthiest neighbourhoods, about half of the children were injured at intersections with high traffic volume or on major arterial roads. The mean number of young pedestrians injured was 39 times greater at intersections with high traffic volume (5 $5^{\text {th }}$ quintile) compared to intersections within the $1^{\text {st }}$ quintile. Locally weighted least square regression analyses show variations in the effect of traffic volume across central boroughs with a similar number of children per square kilometer.
\end{abstract}

\title{
Keywords
}

Injuries, pedestrians, cyclists, children, road safety, public health.

\section{Resumen}

Esta investigación presenta la cuantidad y la distribución geográfica de los niños heridos por un vehículo motorizado en medio urbano y su asociación con factores ambientales conocidos. La investigación se realizó sobre un total de 6775 niños (de 5 a 17 años de edad) heridos en la red de carreteras de la ciudad de Montreal, entre los años 1999 y 2008. Los análisis descriptivos muestran que, dentro los distritos y las municipalidades más pobres como más ricas, cerca de la mitad del total de niños heridos en las esquinas lo fueron en lugares de circulación densa o en rutas importantes. El valor medio de peatones menores heridos es 39 veces más alto en las esquinas con mayor volumen de circulación automóvil (5a quintilla) que en las situadas en la $1^{\text {ra }}$ quintilla. Las regresiones locales ponderadas ilustran la variación del efecto del volumen de circulación en tres distritos centrales similares con una elevada densidad de niños.

\section{Palabras claves}

Heridas, peatones, ciclistas, niños, seguridad vial, salud pública.

\section{Introduction}

Au Canada, les traumatismes routiers et les autres blessures sont la première cause de décès chez les jeunes de 1 à 34 ans (Public Health Agency of Canada, 2005). Au Québec, de 1999 à 2008, plus de 30000 enfants âgés de 5 à 14 ans ont été blessés sur les routes, selon les rapports d'accidents policiers, soit plus de 3000 par an (Société de l'assurance automobile du Québec, 2005 et 2009). Pour près de la moitié (46\%) des piétons et du tiers (32\%) des cyclistes blessés au Québec au cours de cette période, la collision est survenue sur l'île de Montréal.

De nombreuses études portant sur des individus, des populations, des intersections, des quartiers ou des villes ont démontré la grande contribution des volumes de circulation à l'ampleur du bilan routier (Roberts et Crombie, 1995 ; Lyon et Persaud, 2002 ; Ewing et Dumbaugh, 2009; Wier et al., 2009; Miranda-Moreno et al., 2010). Pour améliorer la sécurité des usagers de la route, il est généralement reconnu que 
les stratégies environnementales constituent souvent la stratégie d'intervention la plus efficace (Peek-Asa et Zwerling, 2003; Retting et al., 2003). Plusieurs mesures environnementales liées à la géométrie du réseau routier ont été reconnues efficaces pour réduire le nombre de blessés (Elvik, 2001 ; Ewing et Dumbaugh, 2009; Grundy et al., 2009). Cette recherche vise principalement à décrire le nombre et la distribution géographique des enfants d'âge scolaire blessés à la suite d'une collision routière dans un milieu urbain. Elle vise aussi à explorer l'association entre, d'une part, les caractéristiques des intersections et, d'autre part, le nombre et la répartition des enfants blessés par un véhicule à moteur.

\section{Modèle conceptuel et revue des écrits scientifiques pertinents}

Les modèles conceptuels récents décrivant l'influence de l'environnement bâti sur la sécurité routière distinguent deux niveaux de facteurs environnementaux (Ewing et Dumbaugh, 2009; Miranda-Moreno et al., 2010; Morency, 2010). À l'échelle d'un quartier ou d'une ville, ces modèles identifient la forme urbaine, les caractéristiques du réseau routier (capacité, connexité, etc.), l'offre de transport collectif et le profil démographique des résidants. À l'échelle du réseau routier, ces modèles identifient trois facteurs proximaux ou médiateurs: le volume de circulation automobile, la vitesse des véhicules et les conflits potentiels entre les usagers de la route. Les modèles conceptuels spécifiquement développés pour les piétons ajoutent le volume de déplacements à pied ou de piétons sur le réseau routier (Miranda-Moreno et al., 2010; Morency, 2010). Les prochains paragraphes portent sur des caractéristiques du réseau routier - les facteurs proximaux - identifiées par les modèles conceptuels.

Différents types d'études ont documenté le rôle du volume de véhicules en circulation dans l'épidémiologie des collisions impliquant des jeunes piétons. L'évolution et les fluctuations temporelles du nombre de jeunes piétons blessés ont été associées à l'évolution des volumes de circulation automobile, par exemple aux États-Unis, de 1970 à 1988 (Roberts et Crombie, 1995). Selon des études cas-témoins, le risque pour un jeune piéton d'être blessé par une voiture augmente avec les débits de circulation: par exemple, en Nouvelle-Zélande,ce risque serait 13 fois plus élevé dans les rues ayant un débit égal ou supérieur à 750 véhicules par heure que dans celles où il y a moins de 250 véhicules par heure (Roberts et al., 1995). Aux intersections, la relation entre le nombre de piétons blessés et le volume de circulation automobile a été modélisée par plusieurs études: d'une manière générale, le nombre moyen de piétons blessés est proportionnel à la racine carrée des volumes de circulation automobile aux intersections (Lyon et Persaud, 2002; Garder, 2004 ; Lee et Abdel-Aty, 2005). Cependant, ces études sont limitées aux sites pour lesquels des comptages de véhicules étaient disponibles et ne distinguent pas les jeunes piétons des piétons adultes.

À Edmonton (Canada), la corrélation, à l'échelle des secteurs de recensement, entre la densité de circulation automobile et le taux d'incidence de jeunes piétons blessés est plutôt modérée $(\mathrm{r}=0,38)$ mais cette recherche est limitée par l'utilisation des lieux de résidence des personnes blessées plutôt que des lieux de collision (Yiannakoulias et al., 2002). Faute d'estimés des volumes de circulation automobile, plusieurs études écologiques utilisent des indicateurs indirects tels que la densité d'artères ou d'emplois (Clifton et Kreamer-Fults, 2007; Cloutier et al., 2007). Lorsque l'analyse inclut une mesure des volumes de circulation automobile, cet indicateur est toujours significativement associé au nombre de piétons blessés (La Scala et al., 2004; Wier et al., 2009). 
En plus des débits de circulation plus élevés, les artères comptent généralement davantage de voies de circulation et des chaussées plus larges que les rues collectrices et locales, deux caractéristiques fortement associées à la vitesse des véhicules (Ewing et Dumbaugh, 2009). De plus, le rayon de courbure appliqué aux coins des intersections peut être différent, ce qui est associé à la vitesse de virage des véhicules et à la longueur des passages pour piétons (Association mondiale de la route, 2003). Plusieurs études ont rapporté l'influence des artères et des routes majeures sur le nombre de piétons blessés, mais elles traitent de l'ensemble des piétons et ne distinguent pas les jeunes des adultes. Aux États-Unis, de 1997 à 2006, plus de la moitié des piétons décédés en milieu urbain ont été victimes d'une collision sur une artère (National Highway Traffic Safety Administration, 2008). À Long Beach en Californie, seulement $34 \%$ des piétons blessés ont subi une collision sur une rue locale alors que celles-ci représentent $78 \%$ du réseau routier (Lightstone et al., 2001). Dans le Maine (É.-U.), une étude portant sur 122 traverses piétonnes rapporte que les taux de piétons blessés sont significativement plus élevés sur les routes plus larges, en tenant compte des volumes de véhicules et de piétons (Garder, 2004). Plus récemment, des études écologiques ont rapporté des effets indépendants de la proportion d'artères et du volume estimé de circulation automobile dans un quartier sur le nombre total de blessés ou sur le nombre de piétons blessés (Dumbaugh et Rae, 2009; Wier et al., 2009).

À une intersection constituée de quatre branches, il y a davantage d'occasions de collision, de points de conflit potentiels entre les mouvements des usagers de la route, qu'à une intersection en forme de $\mathrm{T}$, qu'à deux intersections en forme de T rapprochées ou qu'aux ronds-points (Ewing et Dumbaugh, 2009; Federal Highway Administration, 2004). Par conséquent, les taux de collisions et de blessés sont généralement plus élevés aux intersections constituées de quatre branches, pour les piétons comme pour les autres usagers de la route (Lyon et Persaud, 2002; Ewing et Dumbaugh, 2009; Miranda-Moreno et al., 2010). À l'échelle des quartiers, le nombre et la proportion d'intersections à quatre branches serait aussi associés à une incidence plus élevée de collisions et de blessés de la route (Dumbaugh et Rae, 2009).

\section{Recherche basée sur une approche populationnelle}

L'approche populationnelle porte sur les déterminants - les causes sous-jacentes - de l'incidence d'un problème de santé dans l'ensemble d'une population (Rose, 1992). Elle implique la prise en compte de la distribution des problèmes de santé et de leurs déterminants dans la population, incluant le contexte social et la position sociale des individus (Kindig et Stoddart, 2003). La position socioéconomique est généralement associée au risque de blessures (Laflamme et al., 2009). À Montréal (Canada) et dans le département du Rhône (France), les taux de blessures liées aux collisions routières sont plus élevés chez les enfants habitant dans les quartiers les plus pauvres (Dougherty et al., 1990; Licaj, 2011).

Dans le domaine de la prévention des traumatismes routiers, de nombreuses recherches et interventions se limitent aux sites de collision comptant le plus grand nombre de blessés ou à risque élevé de collision, aussi nommés «sites dangereux", «points noirs» ou black spots. Au contraire de l'approche populationnelle, ces approches ciblées (high risk preventive strategy) ne portent que sur une faible fraction des cas (Morency et Cloutier, 2006) et ont un potentiel limité pour réduire le nombre 
total de cas dans l'ensemble de la population (Rose, 1992). Le développement des systèmes d'information géographique (SIG) permet maintenant l'analyse spatiale des collisions routières sans limiter le nombre de sites inclus dans les analyses (Lightstone et al., 2001; Morency et Cloutier, 2006; Pulugurtha et al., 2007). Auparavant, l'analyse spatiale de l'ensemble des sites de collision exigeait un devis de recherche de type écologique, agrégeant les données à l'échelle d'un secteur de recensement, d'un quartier, etc.

La présente recherche s'inspire de l'approche populationnelle et vise principalement à décrire le nombre et la distribution géographique des enfants d'âge scolaire blessés à la suite d'une collision routière dans un milieu urbain. Elle vise aussi à explorer l'association entre, d'une part, le volume de circulation automobile, le type de route et le nombre de branches aux intersections et, d'autre part, le nombre et la répartition des enfants blessés par un véhicule à moteur.

\section{Méthode}

Cette recherche a été réalisée dans la région métropolitaine de Montréal, où vit la moitié (51\%) de la population de la province de Québec (Canada). Montréal est, après Toronto, la deuxième agglomération la plus populeuse du Canada. Plus spécifiquement, l'étude a été réalisée sur l'île de Montréal, un territoire de 499 km² situé au cœur de l'agglomération et qui comptait, en 2006, 1854442 million d'habitants.

\section{Blessés}

La population ciblée est l'ensemble des enfants âgés de 5 à 17 ans blessés à la suite d'une collision routière, pour lesquels il y a eu une intervention ambulancière sur l'île de Montréal entre le 1er janvier 1999 et le 31 juillet 2008. L'information sur les blessés de la route provient des systèmes d'information des services d'urgences ambulanciers, exclusivement dispensés par la Corporation d’Urgences-santé sur l'île de Montréal. Les registres d'Urgences-santé comprennent notamment les coordonnées géographiques $(\mathrm{x}, \mathrm{y})$ des lieux d'intervention, le mécanisme du traumatisme, le type d'usager de la route, des indicateurs de gravité validés, l'âge et le sexe de la personne blessée (Morency et Cloutier, 2006). Les lieux d’intervention ambulancière ont été assignés sur le réseau routier montréalais (géobase de la Ville de Montréal) à l'aide du logiciel ArcInfo (v9.1). Un rayon de $15 \mathrm{~m}$ autour du point central des intersections a été utilisé pour identifier les enfants blessés aux intersections. La longueur du rayon a été choisie afin d'obtenir une proportion de blessés attribués aux intersections correspondant à celle rapportée par la Ville de Montréal, basée sur les rapports policiers d'accidents.

\section{Hiérarchie routière et nombre de branches aux intersections}

En excluant les autoroutes, le réseau routier hiérarchique montréalais distingue quatre type de routes : artères principales, secondaires, collectrices, rues locales. Une jonction spatiale du réseau routier avec des zones tampons (buffers) d'un rayon de 15 m autour des intersections (ArcInfo v9.1) a permis de mesurer deux caractéristiques des intersections: le nombre de branches ainsi que les types de routes présentes. 


\section{Volume de circulation automobile}

Les enquêtes origine-destination (OD) permettent de décrire la mobilité des résidants de la grande région de Montréal lors d'une journée moyenne d'automne et de construire un portrait représentatif des déplacements effectués lors d'une journée type de semaine. Les circonstances spatiotemporelles des déplacements sont recueillies, notamment les points d'origine et de destination (coordonnées $\mathrm{x}, \mathrm{y}$ ), le motif, la séquence détaillée des modes de transport utilisés, l'heure de départ, etc. (Agence métropolitaine du transport, 2000). Un modèle d'affectation des déplacements mesuré par l'enquête OD 1998 a été utilisé afin d'en dériver des trajets plausibles en véhicules privés sur le réseau routier montréalais. Ces résultats individuels sont ensuite agrégés par tronçons afin d'estimer des profils de charge par segment routier. Pour les intersections, les volumes quotidiens de véhicules sur les routes présentes ont été additionnés. Afin d'améliorer le potentiel de généralisation des résultats, les intersections complexes, connectées à un accès autoroutier $(n=258)$ ou ayant des volumes estimés de circulation automobile supérieurs à 80000 véhicules par jour $(n=54)$ ont été exclues. Les analyses incluent 17636 intersections.

\section{Analyses}

Afin de décrire la répartition géographique des blessés, nous avons utilisé le fichier numérique des arrondissements et des municipalités situées sur l'île de Montréal. Le volume de circulation automobile a été catégorisé en quintiles (seuils de 386, 725, 1665 et 7410 véhicules par jour), mais il est aussi traité comme une variable continue dans les régressions locales pondérées (LOESS ; locally weighted least square regression analysis). Les caractéristiques des intersections ont été dichotomisées selon la présence d'une artère (versus seulement des collectrices et rues locales) ou d'une quatrième branche (versus trois branches seulement). Les arrondissements et municipalités ont été regroupés en quintiles, selon la densité d'enfants âgés de 5 à 17 ans par km² (Agglomération de Montréal, 2009), et en terciles, selon le revenu moyen des familles (recensement canadien de 2006).

Pour l'analyse des associations entre le nombre de blessés et les facteurs environnementaux, seuls les enfants blessés aux intersections ont été inclus. Les analyses considèrent la répartition du nombre de jeunes piétons, cyclistes et occupants de véhicules à moteur (OVM) blessés en fonction des caractéristiques des intersections (volume de circulation automobile, hiérarchie routière, nombre de branches) et des arrondissements et municipalités (densité d'enfants, position socioéconomique). Le nombre moyen d'enfants blessés par intersection est aussi analysé en fonction des caractéristiques des intersections et des arrondissements et municipalités. L'analyse de variance (ANOVA) est utilisée pour vérifier la présence de différences statistiquement significatives et, le cas échéant, la linéarité de la tendance est examinée.

La régression locale pondérée (LOESS), une méthode d’analyse non paramétrique, produit des régressions spécifiques basées sur le voisinage de chaque valeur prédictive, ce qui permet de visualiser, dans un diagramme de dispersion (scatter plot), la forme d'une relation entre deux variables quantitatives sans imposer une relation linéaire, quadratique ou logarithmique (Cleveland et Devlin, 1988). Dans cette recherche, les LOESS permettent, dans un premier temps, d'explorer la forme de l'association 
entre le nombre de jeunes piétons, cyclistes et OVM blessés aux intersections et le volume de circulation automobile. Dans un deuxième temps, ils permettent de décrire d'éventuelles variations selon l'arrondissement ou la municipalité. Les bornes définies par défaut par Stata/SE 9.2 (bandwith=0,8) ont été employées pour sélectionner les données de voisinage utilisées dans les régressions, mais l'usage de bornes inférieures $(0,4-0,8)$ ne modifie pas les résultats de manière significative. Les autres analyses ont été réalisées avec les logiciels SPSS (version 11) et ArcGIS (v9.1).

\section{Résultats}

Au cours de la période étudiée, 6775 enfants âgés de 5 à 17 ans ont été blessés sur le réseau routier montréalais (1799 piétons; 1972 cyclistes; 3004 passagers de VM). Près de la moitié ( $45 \%$ ) des blessés étaient âgés de 5 à 11 ans (49\% des piétons ; $45 \%$ des cyclistes ; $44 \%$ des OVM). Globalement, $57 \%$ étaient de sexe masculin, mais cette proportion varie beaucoup selon le type d'usager de la route (57\% des piétons; 76 des cyclistes ; $43 \%$ des OVM).

Le tableau 1 détaille le nombre d'enfants blessés ainsi que le taux per capita de jeunes piétons et cyclistes blessés sur le territoire d'un arrondissement ou d'une municipalité. Les arrondissements Villeray-Saint-Michel-Parc-Extension (628 blessés) et Mercier-Hochelaga-Maisonneuve (626 blessés) comptent le plus grand nombre d'enfants blessés sur leur territoire. Le taux le plus élevé de jeunes piétons et cyclistes blessés est observé dans l'arrondissement Ville-Marie, suivi de Mercier-HochelagaMaisonneuve (respectivement 390 et 284 jeunes piétons et cyclistes blessés par an pour 100000 enfants).

Les 6775 enfants âgés de 5 à 17 ans qui ont été blessés l'ont été à un grand nombre (4859) de sites différents (figure 1). Pour plus de la moitié (59\%) des enfants blessés, la collision est survenue à une intersection, une proportion relativement similaire pour chaque type d'usager de la route (61\% pour les piétons, $57 \%$ pour les cyclistes, $58 \%$ pour les automobilistes). Seulement $13 \%$ des 2483 intersections touchées comptent plus de deux enfants blessés au cours de la période étudiée (respectivement $5 \%$ pour les piétons, $2 \%$ pour les cyclistes et $13 \%$ pour les OVM). Pour deux tiers (66\%) de tous les enfants blessés aux intersections, la collision est survenue aux sites ne comptant qu'un ou deux blessés (respectivement $86 \%$ pour les piétons, $95 \%$ pour les cyclistes et $70 \%$ pour les OVM).

\section{Répartition des enfants blessés}

Près de quatre enfants sur cinq (79\%) ont été blessés aux intersections ayant un volume de circulation élevé ou très élevé ( 4 e et $5^{\mathrm{e}}$ quintiles) ( $81 \%$ des jeunes piétons, $67 \%$ des jeunes cyclistes, $86 \%$ des jeunes OVM) (tableau 2). Bien que les intersections avec artère ne représentent que $18 \%$ de l'ensemble des intersections, respectivement $53 \%$ des jeunes piétons et $65 \%$ des jeunes OVM ont été blessés à ces intersections. Les intersections ayant quatre branches ne représentent que $41 \%$ de l'ensemble des intersections mais, globalement, quatre enfants sur cinq (80\%) y ont été blessés. 
Tableau 1 Nombre et taux d'enfants blessés dans chacun des arrondissements et municipalités de l'île de Montréal, selon le type d'usager de la route (1999-2008)

\begin{tabular}{|c|c|c|c|c|c|c|}
\hline & \multicolumn{4}{|c|}{ Nombre de blessés } & \multicolumn{2}{|c|}{$\begin{array}{l}\text { Taux annuel de blessés } \\
\text { (/100 } 000 \text { enfants })\end{array}$} \\
\hline & $\begin{array}{c}\text { Occupants de } \\
\text { véhicules motorisés }\end{array}$ & Piétons & Cyclistes & Total & Piétons & Cyclistes \\
\hline Hors arrondissements* & 5 & 0 & 2 & 7 & & \\
\hline Senneville & 1 & 0 & 0 & 1 & 0 & 0 \\
\hline Baie D'Urfé & 10 & 1 & 1 & 12 & 13 & 13 \\
\hline Sainte-Anne-de-Bellevue & 10 & 1 & 3 & 14 & 12 & 37 \\
\hline Hampstead & 9 & 3 & 3 & 15 & 23 & 23 \\
\hline Montréal-Ouest & 6 & 5 & 6 & 17 & 49 & 59 \\
\hline Beaconsfield & 27 & 1 & 11 & 39 & 3 & 29 \\
\hline Dorval & 26 & 5 & 9 & 40 & 20 & 35 \\
\hline Westmount & 20 & 14 & 12 & 46 & 48 & 41 \\
\hline $\begin{array}{l}\text { Île-Bizard- } \\
\text { Sainte-Geneviève }\end{array}$ & 18 & 11 & 18 & 47 & 33 & 55 \\
\hline Montréal-Est & 38 & 4 & 6 & 48 & 68 & 103 \\
\hline Côte Saint-Luc & 24 & 11 & 17 & 52 & 27 & 42 \\
\hline Kirkland & 34 & 8 & 17 & 59 & 19 & 40 \\
\hline Outremont & 14 & 20 & 28 & 62 & 54 & 75 \\
\hline Mont-Royal & 65 & 9 & 14 & 88 & 26 & 41 \\
\hline Pointe-Claire & 76 & 10 & 22 & 108 & 20 & 44 \\
\hline Dollard-des-Ormeaux & 54 & 29 & 43 & 126 & 34 & 50 \\
\hline Lachine & 43 & 33 & 63 & 139 & 58 & 111 \\
\hline Anjou & 97 & 24 & 46 & 167 & 47 & 91 \\
\hline Verdun & 36 & 66 & 91 & 193 & 89 & 123 \\
\hline Pierrefonds-Roxboro & 96 & 43 & 59 & 198 & 37 & 51 \\
\hline LaSalle & 90 & 48 & 83 & 221 & 50 & 87 \\
\hline Plateau-Mont-Royal & 100 & 93 & 66 & 259 & 127 & 90 \\
\hline Saint-Léonard & 144 & 76 & 64 & 284 & 82 & 69 \\
\hline Saint-Laurent & 163 & 74 & 68 & 305 & 62 & 57 \\
\hline Le Sud-Ouest & 147 & 88 & 113 & 348 & 99 & 128 \\
\hline Ville-Marie & 186 & 88 & 94 & 368 & 188 & 201 \\
\hline $\begin{array}{l}\text { Rivière-des-Prairies- } \\
\text { Pointe-aux-Trembles }\end{array}$ & 218 & 84 & 111 & 413 & 51 & 67 \\
\hline Ahuntsic-Cartierville & 200 & 118 & 100 & 418 & 77 & 65 \\
\hline Montréal-Nord & 169 & 142 & 130 & 441 & 117 & 107 \\
\hline $\begin{array}{l}\text { Côte-des-Neiges- } \\
\text { Notre-Dame-de-Grâce }\end{array}$ & 189 & 151 & 138 & 478 & 72 & 66 \\
\hline Rosemont-La Petite-Patrie & 200 & 146 & 162 & 508 & 112 & 124 \\
\hline $\begin{array}{l}\text { Mercier- } \\
\text { Hochelaga-Maisonneuve }\end{array}$ & 221 & 188 & 217 & 626 & 132 & 152 \\
\hline $\begin{array}{l}\text { Villeray-Saint-Michel- } \\
\text { Parc-Extension }\end{array}$ & 268 & 205 & 155 & 628 & 109 & 83 \\
\hline Île de Montréal & 3004 & 1799 & 1972 & 6775 & 75 & 83 \\
\hline
\end{tabular}

* Sept blessés n'ont été attribués à aucun arrondissement ou municipalité (ex. ponts desservant l'île de Montréal). Les taux per capita de jeunes occupants de véhicules à moteur n’ont pas été calculés, car il est plus probable que le lieu de collision ne soit pas situé à proximité du lieu de résidence. 


\section{Figure 1 Cartographie des sites de collision avec au moins un jeune piéton ou cycliste blessé (île de Montréal, 1999-2008)}

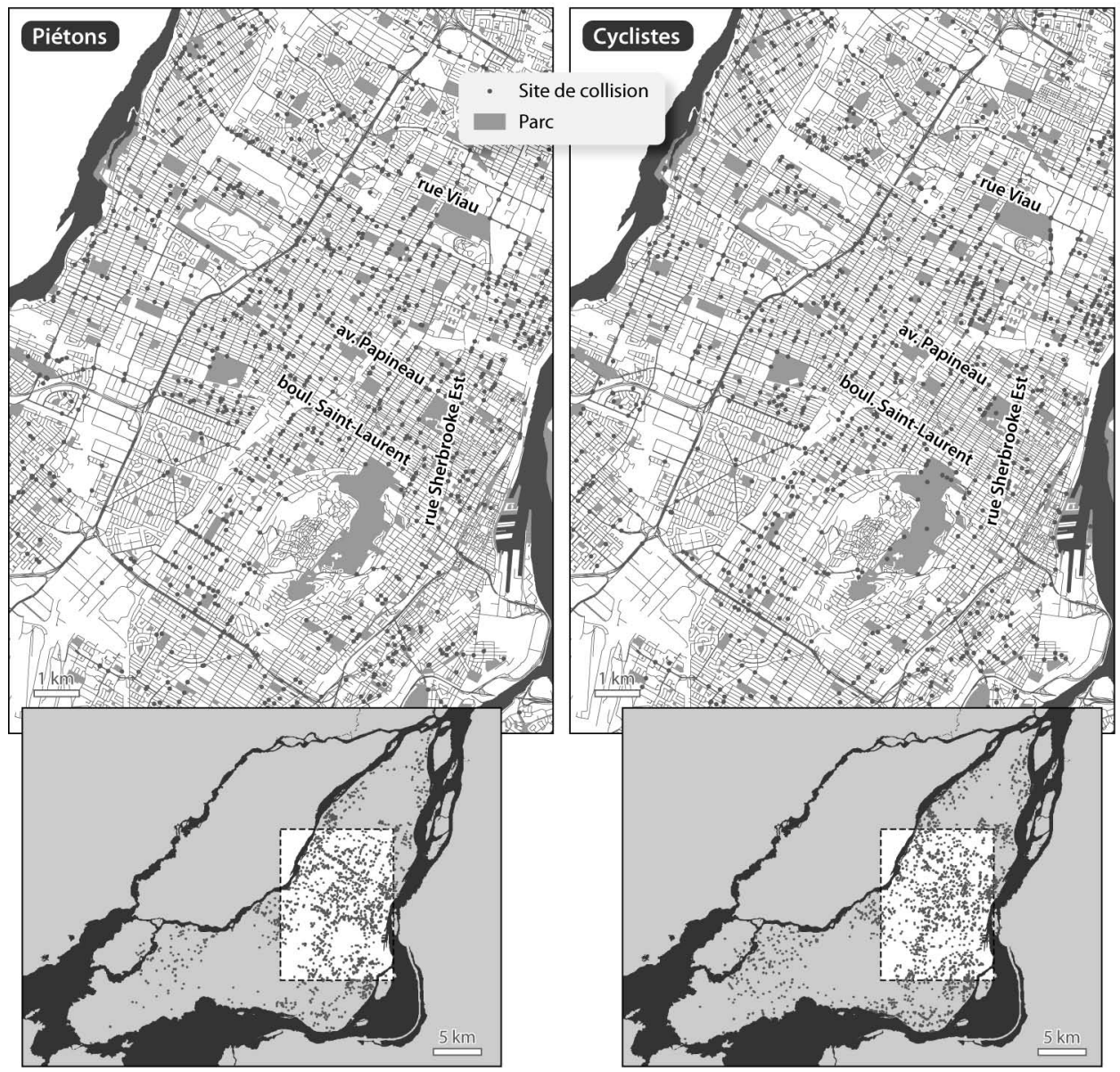

Sur l'île de Montréal, près du tiers (31,9\%) des enfants âgés de 5 à 17 ans habitent dans les six arrondissements et municipalités ayant une densité d'enfants très élevée ( $5^{\mathrm{e}}$ quintile), tandis que seulement $3,4 \%$ des enfants habitent dans les six arrondissements et municipalités ayant une densité d'enfants très faible (1 er $^{\text {quintile). Cette }}$ répartition de la population se reflète dans la distribution des enfants blessés, puisque davantage d'enfants (39\%) ont été blessés dans les arrondissements et municipalités ayant une densité d'enfants très élevée que dans ceux ayant une densité d'enfants très faible (2\%) (tableau 2). De la même façon, la moitié $(50 \%)$ des enfants âgés de 5 à 17 ans habitent dans les 11 arrondissements et municipalités les plus pauvres (1 $1^{\text {er }}$ tercile), où ont été blessés $60 \%$ des enfants blessés sur l'île de Montréal (tableau 2). 
Tableau 2 Répartition des enfants blessés (1999-2008) aux intersections de l'île de Montréal selon le type d'usager de la route et selon les caractéristiques des intersections et des arrondissements et municipalités (île de Montréal)

\begin{tabular}{|c|c|c|c|c|c|c|c|c|c|}
\hline \multirow[b]{3}{*}{ Intersections } & & \multicolumn{8}{|c|}{ Nombre d'enfants blessés } \\
\hline & & \multicolumn{2}{|c|}{ Piétons } & \multicolumn{2}{|c|}{ Cyclistes } & \multicolumn{2}{|c|}{$\begin{array}{c}\text { Occupants de } \\
\text { véhicules motorisés }\end{array}$} & \multicolumn{2}{|c|}{ Tous } \\
\hline & & $\mathbf{n}$ & $\%$ & $\mathbf{n}$ & $\%$ & $\mathbf{n}$ & $\%$ & n & $\%$ \\
\hline \multirow{6}{*}{$\begin{array}{l}\text { Volume de circulation } \\
\text { automobile (quintiles) }\end{array}$} & Très faible (1 $\left.{ }^{\mathrm{er}}\right)$ & 16 & 2 & 71 & 6 & 40 & 2 & 127 & 3 \\
\hline & Faible $\left(2^{\mathrm{e}}\right)$ & 64 & 6 & 123 & 11 & 77 & 5 & 264 & 7 \\
\hline & Moyen $\left(3^{\mathrm{e}}\right)$ & 118 & 11 & 176 & 16 & 109 & 7 & 403 & 11 \\
\hline & Élevé (4e) & 236 & 22 & 279 & 25 & 359 & 22 & 874 & 23 \\
\hline & Très élevé (5e) & 626 & 59 & 464 & 42 & 1024 & 64 & 2114 & 56 \\
\hline & & & $100 \%$ & & $100 \%$ & & $100 \%$ & & $100 \%$ \\
\hline \multirow{3}{*}{ Présence d'artère } & Non & 494 & 47 & 743 & 67 & 556 & 35 & 1793 & 47 \\
\hline & Oui & 566 & 53 & 370 & 33 & 1053 & 65 & 1989 & 53 \\
\hline & & & $\overline{100 \%}$ & & $\overline{100 \%}$ & & $\overline{100 \%}$ & & $\overline{100 \%}$ \\
\hline \multirow{3}{*}{$\begin{array}{l}\text { Présence de quatre } \\
\text { branches }\end{array}$} & Non & 204 & 19 & 307 & 28 & 232 & 14 & 743 & 20 \\
\hline & Oui & 856 & 81 & 806 & 72 & 1377 & 86 & 3039 & 80 \\
\hline & & & $\overline{100 \%}$ & & $\overline{100 \%}$ & & $\overline{100 \%}$ & & $\overline{100 \%}$ \\
\hline \multicolumn{10}{|c|}{ Arrondissements et municipalités } \\
\hline \multirow{6}{*}{$\begin{array}{l}\text { Densité d'enfants âgés } \\
\text { de } 5 \text { à } 17 \text { ans } \\
\text { (quintiles) }\end{array}$} & Très faible $\left(1^{\mathrm{er}}\right)$ & 7 & 1 & 21 & 2 & 29 & 2 & 57 & 2 \\
\hline & Faible $\left(2^{\mathrm{e}}\right)$ & 174 & 16 & 232 & 21 & 356 & 22 & 762 & 20 \\
\hline & Moyenne $\left(3^{\mathrm{e}}\right)$ & 242 & 23 & 310 & 28 & 383 & 24 & 935 & 25 \\
\hline & Élevée (4e) & 164 & 15 & 177 & 16 & 218 & 14 & 559 & 15 \\
\hline & Très élevée ( $5^{\mathrm{e}}$ ) & 473 & 45 & 373 & 34 & 623 & 39 & 1469 & 39 \\
\hline & & & $100 \%$ & & $100 \%$ & & $100 \%$ & & $100 \%$ \\
\hline \multirow{4}{*}{ Revenu familial moyen } & Riche & 41 & 4 & 71 & 6 & 117 & 7 & 229 & 6 \\
\hline & Moyen & 341 & 32 & 366 & 33 & 576 & 36 & 1283 & 34 \\
\hline & Pauvre & 678 & 64 & 676 & 61 & 916 & 57 & 2270 & 60 \\
\hline & & & $\overline{100 \%}$ & & $\overline{100 \%}$ & & $\overline{100 \%}$ & & $\overline{100 \%}$ \\
\hline
\end{tabular}


Figure 2 Répartition des enfants blessés aux intersections selon la position socioéconomique des arrondissements et municipalités et les caractéristiques des intersections (île de Montréal, 1999-2008)

a) Volume de circulation automobile (quintiles)

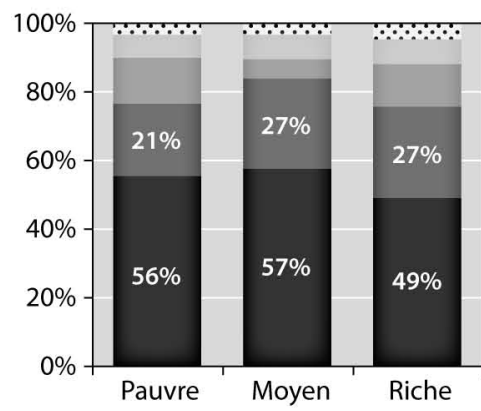

Revenu familial moyen (tercile) des arrondissements et municipalités

b) Hiérarchie routière

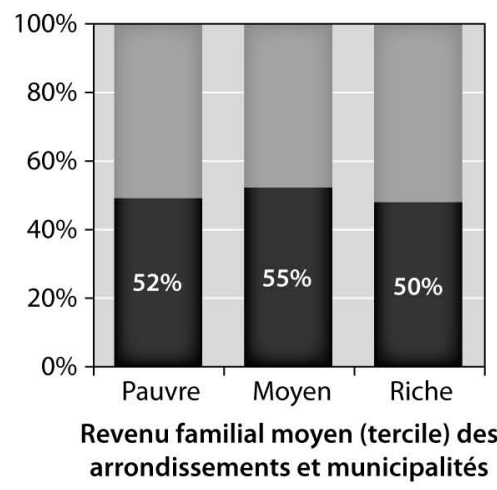

c) Nombre de branches

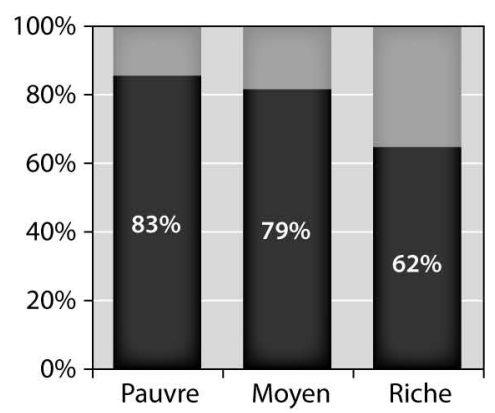

Revenu familial moyen (tercile) des arrondissements et municipalités
La figure 2 illustre la répartition des enfants blessés selon la position socioéconomique des arrondissements et municipalités et les caractéristiques des intersections. Dans les secteurs pauvres comme dans les secteur riches de l'île de Montréal, plus des trois quarts des enfants blessés aux intersections l'ont été à des sites ayant un volume de circulation élevé ou très élevé $(76 \%$ à $84 \%)$ et environ la moitié l'ont été à des intersections avec artère (50\% à $55 \%)$. Dans les arrondissements et municipalités les plus riches, la proportion d'enfants blessés aux intersections à quatre branches (62\%) est légèrement inférieure à celle observée ailleurs sur l'île de Montréal.

\section{Nombre moyen d'enfants blessés aux intersections}

L'association entre le nombre moyen d'enfants blessés aux intersections et le quintile de volume de circulation automobile, la présence d'une artère et d'une quatrième branche est statistiquement significative pour les trois types d'usagers de la route $(\mathrm{p}<0,001)$. Par exemple, il y a 39 fois plus de piétons, 7 fois plus de cyclistes et 26 fois plus d'OVM blessés aux intersections situées dans le cinquième quintile de circulation automobile (très élevée) qu'aux intersections situées dans le premier quintile (tableau 3 ). L'association entre le nombre moyen d'enfants blessés aux intersections et les caractéristiques des arrondissements 
(densité d'enfants, revenu familial moyen) est statistiquement significative pour les trois types d'usagers de la route $(\mathrm{p}<0,001)$. Il y a davantage d'enfants blessés aux intersections des arrondissements et municipalités ayant une densité d'enfants plus élevée et/ou un revenu familial plus faible (tableau 3).

Tableau 3 Nombre moyen d'enfants blessés (1999-2008) à 100 intersections selon le type d'usager de la route et selon les caractéristiques des intersections et des arrondissements et municipalités (île de Montréal)

\begin{tabular}{|c|c|c|c|c|c|}
\hline & \multirow[b]{3}{*}{ Île de Montréal } & \multicolumn{4}{|c|}{ Nombre moyen d'enfants blessés à 100 intersections } \\
\hline & & Piétons & Cyclistes & $\begin{array}{c}\text { Occupants } \\
\text { de véhicules } \\
\text { motorisés }\end{array}$ & Tous \\
\hline & & 6,0 & 6,3 & 9,1 & 21,4 \\
\hline \multicolumn{6}{|l|}{ Intersections } \\
\hline \multirow{6}{*}{$\begin{array}{l}\text { Volume de } \\
\text { circulation } \\
\text { automobile } \\
\text { (quintiles) }\end{array}$} & Très faible ( $\left.1^{\text {er }}\right)$ & 0,5 & 2,0 & 1,1 & 3,6 \\
\hline & Faible $\left(2^{\mathrm{e}}\right)$ & 1,8 & 3,5 & 2,2 & 7,5 \\
\hline & Moyen $\left(3^{\mathrm{e}}\right)$ & 3,3 & 5,0 & 3,1 & 11,4 \\
\hline & Élevé (4e) & 6,7 & 7,9 & 10,2 & 24,8 \\
\hline & Très élevé ( $\left.5^{\mathrm{e}}\right)$ & 17,7 & 13,2 & 29,0 & 59,9 \\
\hline & Ratio: $5^{\mathrm{e}} / 1^{\mathrm{er}}$ quintile & 39,1 & 6,5 & 25,6 & 16,6 \\
\hline \multirow{3}{*}{ Présence d'artère } & Non & 3,4 & 5,1 & 3,8 & 12,3 \\
\hline & Oui & 18,3 & 12,0 & 34,1 & 64,4 \\
\hline & Ratio: Avec / sans artère & 5,4 & 2,3 & 8,9 & 5,2 \\
\hline \multirow{3}{*}{$\begin{array}{l}\text { Présence de quatre } \\
\text { branches }\end{array}$} & Non & 2,0 & 2,9 & 2,2 & 7,1 \\
\hline & Oui & 11,9 & 11,2 & 19,1 & 42,3 \\
\hline & Ratio: Avec / sans $4^{\mathrm{e}}$ branche & 6,1 & 3,8 & 8,6 & 5,9 \\
\hline \multicolumn{6}{|c|}{ Arrondissements et municipalités } \\
\hline \multirow{6}{*}{$\begin{array}{l}\text { Densité d'enfants } \\
\text { âgés de } 5 \text { à } 17 \text { ans } \\
\text { (quintiles) }\end{array}$} & Très faible ( $\left.1^{\text {er }}\right)$ & 0,7 & 2,0 & 2,7 & 5,3 \\
\hline & Faible $\left(2^{\mathrm{e}}\right)$ & 3,5 & 4,6 & 7,1 & 15,1 \\
\hline & Moyenne $\left(3^{\mathrm{e}}\right)$ & 4,9 & 6,3 & 7,8 & 19,1 \\
\hline & Élevée (4e) & 5,9 & 6,3 & 7,8 & 19,9 \\
\hline & Très élevée $\left(5^{\mathrm{e}}\right)$ & 12,4 & 9,7 & 16,3 & 38,4 \\
\hline & Ratio: $5^{\mathrm{e}} / 1^{\mathrm{er}}$ quintile & 19,0 & 5,0 & 6,0 & 7,2 \\
\hline \multirow{4}{*}{$\begin{array}{l}\text { Revenu familial } \\
\text { moyen }\end{array}$} & Riche & 1,5 & 2,6 & 4,2 & 8,3 \\
\hline & Moyen & 4,5 & 4,8 & 7,5 & 16,8 \\
\hline & Pauvre & 9,4 & 9,4 & 12,7 & 31,5 \\
\hline & Ratio: Pauvre / Riche & 6,4 & 3,7 & 3,0 & 3,8 \\
\hline
\end{tabular}


Figure 3 Régressions locales pondérées du nombre d'enfants blessés aux intersections en fonction du volume de circulation automobile, pour trois arrondissements montréalais ayant une densité très élevée d'enfants âgés de 5 à 17 ans

a) Piétons et cyclistes

- Montréal-Nord

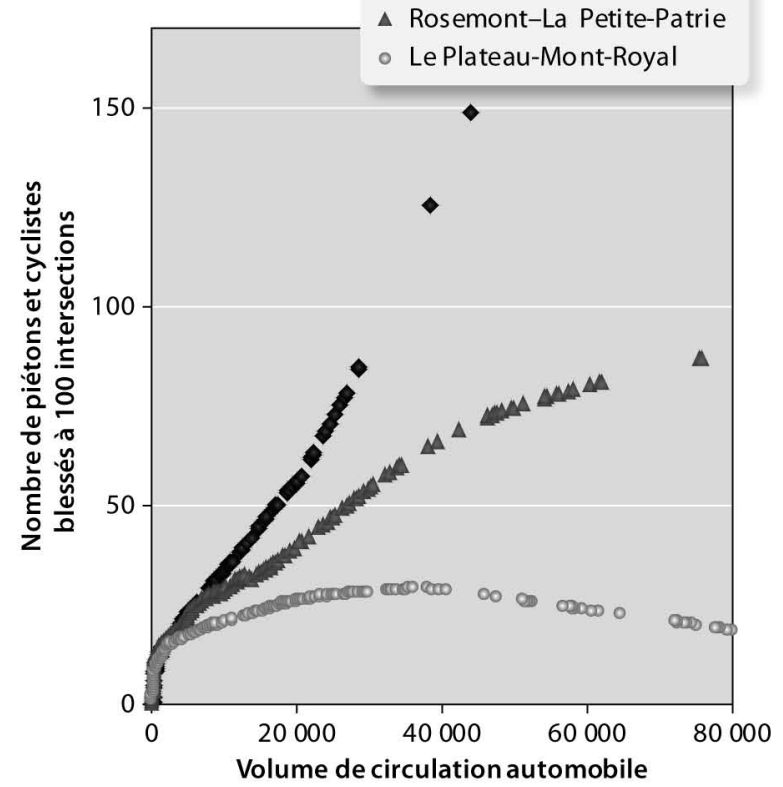

b) Occupants de véhicules à moteur

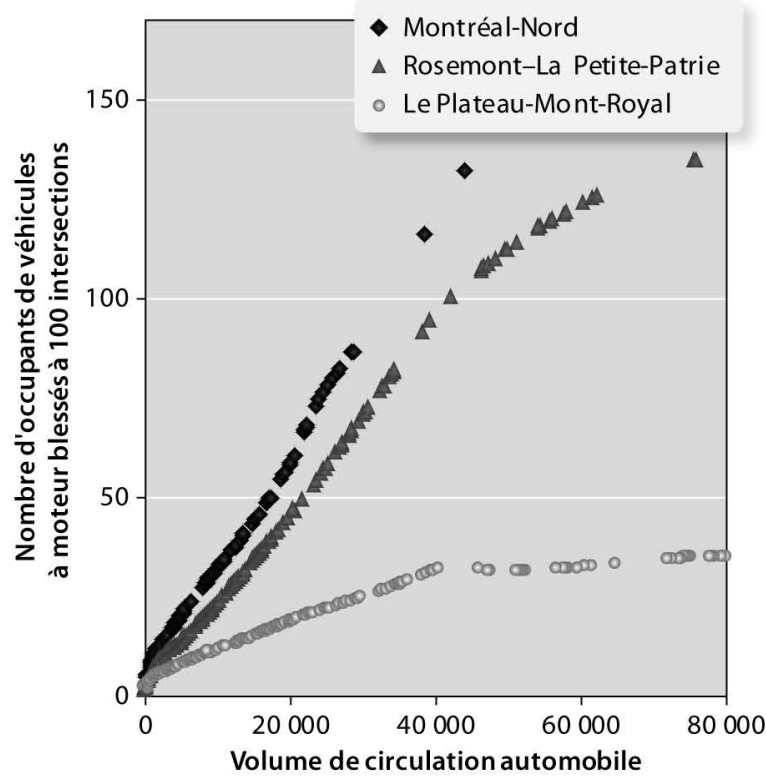

Variation spatiale de l'effet du volume de circulation

La figure 3 illustre la forme de l'association entre le nombre d'enfants blessés aux intersections et le volume de circulation automobile pour trois arrondissements montréalais. Les arrondissements Montréal-Nord, Rosemont-La Petite-Patrie et Le Plateau-Mont-Royal ont été choisis parce qu'ils ont une densité d'enfants très élevée (5e quintile) et relativement similaire (respectivement 1143, 86 et 940 enfants âgés de 5 à 17 ans par $\mathrm{km}^{2}$ ). D’une manière générale, le nombre moyen d'enfants blessés aux intersections tend à augmenter avec le volume de circulation. Cependant, à volume de circulation égal, le nombre moyen d'enfants blessés semble plus faible dans l'arrondissement Le Plateau-Mont-Royal, tant pour les piétons et les cyclistes (figure 3a) que pour les occupants de véhicules à moteur (figure 3b). Enfin, la pente - l'augmentation du nombre moyen de blessés associé à une augmentation de la circulation automobile - semble beaucoup plus prononcée dans les arrondissements Rosemont-La PetitePatrie et Montréal-Nord que dans Le Plateau-Mont-Royal.

* La densité d'enfants âgés de 5 à 17 ans par km² est de 1143 dans Montréal-Nord, de 860 dans Rosemont-La Petite-Patrie et de 940 dans Le Plateau-Mont-Royal. 


\section{Discussion}

Inspirée d'une approche populationnelle, cette recherche a permis de décrire le nombre et la distribution géographique des enfants d'âge scolaire blessés à la suite d'une collision routière dans un milieu urbain, en fonction des caractéristiques des intersections (volume de circulation automobile, hiérarchie routière, nombre de branches aux intersections) et des arrondissements et municipalités (densité d'enfants, position socioéconomique).

\section{Nombre d'enfants blessés}

Les résultats confirment le grand nombre d'enfants blessés sur le réseau routier montréalais. Dans les arrondissements centraux adjacents au centre-ville (Le Plateau-Mont-Royal, Rosemont-La Petite-Patrie, Mercier-Hochelaga-Maisonneuve, SudOuest) sur une période de 10 ans, le nombre de jeunes piétons et de cyclistes blessés représente jusqu'à $2 \%$ ou $3 \%$ des enfants habitant dans l'arrondissement. Le nombre réel d'enfants blessés est sans doute encore plus élevé, puisque cette recherche n'a utilisé qu'une seule source d'information, qui n'inclut pas les collisions et les blessés pour lesquels il n’y a pas eu d'intervention ambulancière. Pour la période 1999-2007, le nombre de piétons et de cyclistes blessés sur l'île de Montréal, selon les registres d'interventions ambulancières représente environ les trois quarts (77\%) du nombre de blessés comptés par les rapports d'accidents policiers (Société de l'assurance automobile du Québec, 2005 et 2009). D’autres études ont utilisé les rapports policiers (Clifton et Kreamer-Fults, 2007; Cloutier et al., 2007), les fichiers d'indemnisation (Schofield et al., 2008) ou les registres hospitaliers (Dougherty et al., 1990), mais aucune n'a intégré les différentes sources de données disponibles sur les traumatismes routiers afin de mieux estimer le nombre total d'enfants blessés dans une ville. C'est une piste de recherche à exploiter dans le futur et qui pourrait soutenir la création d'un système de surveillance des blessés de la route.

\section{Nombre de sites de collisions}

L’analyse désagrégée a permis de mettre en évidence le grand nombre de sites de collisions différents ainsi que leur dispersion au sein des quartiers (figure 1). Les études écologiques, agrégeant les sites de collision dans un quartier ou autour d'une école (Lascala et al., 2004 ; Clifton et Kreamer-Fults, 2007 ; Cloutier et al., 2007), ne peuvent pas ainsi décrire la distribution des blessés et des sites de collision sur le réseau routier. Bien que cette recherche inclue près de 4000 enfants blessés aux intersections, les intersections comptant plus de deux enfants blessés ne représentent qu'une minorité des sites de collision (13\%) et des enfants blessés (34\%). Ce phénomène a déjà été décrit pour l'ensemble des piétons blessés (Morency et Cloutier, 2006) ; il illustre l'une des limites des approches préventives ne ciblant que les sites comptant le plus grand nombre de blessés.

\section{Répartition des enfants blessés}

De nombreuses recherches portent sur la quantification du risque de collision et de blessures associées à une caractéristique environnementale, à partir de devis de recherche écologique, de cas-témoins ou se limitant aux sites pour lesquels des 
comptages sont disponibles. Aucun de ces devis ne permet de décrire la répartition de l'ensemble des enfants blessés sur le réseau routier. Sur l'île de Montréal, la collision survient sur les routes à forte circulation automobile ou sur les artères pour une part substantielle des enfants blessés. Ce constat s'applique aux trois types d'usagers de la route étudiés, aux arrondissements et municipalités les plus pauvres comme aux plus riches (tableau 2 et figure 2).

\section{Influence des facteurs environnementaux}

Cette recherche transversale est principalement descriptive. Des devis expérimentaux ou quasi expérimentaux seraient requis pour confirmer l'existence d'un lien causal entre les facteurs environnementaux étudiés et le nombre d'enfants blessés. Cependant, les mécanismes par lesquels un volume de circulation élevé et la géométrie des routes, liée à la hiérarchie routière et au nombre de branches, peuvent accroître le risque de collision sont bien connus (Association mondiale de la route, 2003 ; Federal Highway Administration, 2004) et inclus dans les modèles conceptuels développés récemment (Ewing et Dumbaugh, 2009; Miranda-Moreno et al., 2010). Il y a donc une explication causale plausible pour expliquer la présence des associations observées. Dans cette recherche, le nombre moyen d'enfants blessés est considérablement plus élevé aux intersections à volume élevé de circulation automobile, ainsi qu'aux intersections avec une artère ou dotés d'une quatrième branche (tableau 3).

La densité d'enfants est un indicateur indirect du nombre de jeunes piétons exposés au risque de collision, mais elle ne permet pas de connaître le nombre et la répartition des piétons sur le réseau routier. Ainsi, dans notre recherche, on ne peut exclure que le volume de piétons aux intersections explique une partie de l'excès de piétons blessés observé aux intersections ayant une forte circulation automobile, avec une artère ou dotés d'une quatrième branche. Le cas échéant, cela impliquerait qu'il y a davantage de jeunes piétons à ces intersections, ce qui représenterait un certain paradoxe pour la santé publique, notamment pour les programmes de promotion de la marche et du vélo chez les jeunes.

Les régressions locales pondérées (figure 3 ) illustrent une variation de l'effet du volume de circulation sur le nombre de blessés selon l'arrondissement, pour trois arrondissements ayant une densité d'enfants élevée et relativement similaire. Il est tout à fait plausible que cet effet varie au sein d'une ville, par exemple en fonction de la qualité et de la sécurité des aménagements routiers. Deux études anglaises ont montré que l'implantation des mesures d'apaisement de la circulation n'est pas uniforme sur le territoire (Lyons et al., 2006; Rodgers et al., 2010). Les modèles de régression habituels ne permettent pas de prendre en compte la variation spatiale de l'effet du volume de circulation sur le nombre de blessés. Des recherches ultérieures pourraient utiliser des modèles de régression multiniveaux ou des régressions géographiques pondérées (GWR), qui seraient plus appropriés.

\section{Forces}

Au contraire des études écologiques, cette recherche n’agrège pas les sites de collision et les caractéristiques environnementales. L'analyse du lien entre le nombre de blessés et les caractéristiques des intersections se fait au niveau de l'intersection, ce qui 
permet d'éliminer une possible erreur écologique (Diez-Roux, 1998). De plus, cette recherche considère l'ensemble des sites potentiels de collision, sans égard au nombre ou aux caractéristiques des enfants blessés, sans égard à la présence ou à l'intensité des facteurs environnementaux étudiés. Elle ne se limite donc pas à un échantillon de sites routiers ou de blessés. Enfin, les dimensions étudiées comprennent le volume de circulation automobile - un facteur fondamental - ainsi que la hiérarchie routière et le nombre de branches, qui reflètent deux dimensions majeures et distinctes du risque de collision: d'une part, les aménagements augmentant la capacité routière et la vitesse des véhicules et, d'autre part, le nombre de points de conflit entre les usagers de la route.

\section{Limites}

Le devis transversal ne permet pas de prendre en compte l'évolution temporelle des facteurs environnementaux, ni de démontrer l'antériorité des facteurs environnementaux sur l'occurrence des collisions et des blessés. L'utilisation du revenu familial moyen comme indicateur de la position socioéconomique des arrondissements et des municipalités ne permet pas de connaître les ressources réelles des administrations locales, ni la position socioéconomique des enfants blessés. Les enquêtes originedestination ne permettent pas de prendre en compte le volume de camionnage sur le réseau routier. D’autres sources de données pourraient être utilisées pour améliorer la mesure des facteurs environnementaux. Certaines associations observées pourraient être validées avec des comptages de véhicules, la source habituelle de données pour les études à l'échelle des intersections. La mesure de la géométrie des routes et des intersections pourrait être plus détaillée. L'association entre les facteurs environnementaux et le nombre d'enfants blessés sur les tronçons routiers, entre les intersections, n’est pas étudiée. Une prochaine recherche pourrait prendre en compte les volumes estimés de piétons et de cyclistes et, tel que mentionné ci-haut, utiliser des analyses de régression multiniveaux ou géographiquement pondérées. Des analyses multivariées seraient requises pour développer un modèle explicatif, étiologique, ou démontrer l'effet indépendant de chacun des facteurs environnementaux.

\section{Conclusion}

Cette recherche a permis de décrire le nombre et la distribution géographique des enfants blessés par un véhicule à moteur en milieu urbain, ainsi que l'association avec des facteurs environnementaux liés au réseau routier. Le volume de circulation automobile, la hiérarchie routière et le nombre de branches aux intersections semblent avoir une large incidence sur le nombre d'enfants blessés, tant pour les piétons que pour les cyclistes et les occupants de véhicules à moteur. Les résultats confirment la pertinence des stratégies préventives de nature environnementale, telles que la réduction des volumes de circulation automobile et l'implantation de mesures d'apaisement de la circulation, surtout dans un contexte de promotion de l'usage de la marche et du vélo chez les jeunes. 


\section{Remerciements}

Nous aimerions remercier le personnel d'Urgences-santé, en particulier Éric Lareau, pour l'excellente collaboration dans l'obtention et la validation des données sur les blessés de la route. Cette recherche a bénéficié du soutien financier du Programme de subventions en santé publique. Lise Gauvin est titulaire de la Chaire IRSC-CRPO (Instituts de recherche en santé du Canada-Centre de recherche en prévention de l'obésité) en santé publique appliquée sur les voisinages, les habitudes de vie et le poids santé. Catherine Morency est titulaire de la Chaire de recherche sur l'évaluation et la mise en œuvre de la mobilité durable. Patrick Morency est professeur adjoint de clinique au Département de médecine sociale et préventive de l'Université de Monréal. Parick Morency et Lise Gauvin sont affiliés au Centre de recherche du Centre Hospitalier de l'Université de Montréal (CRCHUM). Luis Miranda-Moreno et Catherine Morency sont membres du Centre interuniversitaire de recherche sur les réseaux d'entreprise, la logistique et le transport (CIRRELT). Patrick Morency, Marie-Soleil Cloutier et Luis Miranda-Moreno sont membres du réseau stratégique de recherche et d'innovation en sécurité routière financée par le Fonds québécois de la recherche sur la nature et les technologies (FQRNT). 


\section{Bibliographie}

AGENCE MÉTROPOLITAINE DU TRANSPORT (2000) Enquête origine-destination 1998, la mobilité des personnes dans la région de Montréal.

AGGLOMÉRATION DE MONTRÉAL (2009) Profils sociodémographiques (2006) des arrondissements et villes reconstituées. [En ligne.] http://www.ville.montreal.qc.ca/ montrealenstatistiques

ASSOCIATION MONDIALE DE LA ROUTE (AIPCR) (2003) Manuel de la sécurité routière.

CLOUTIER, Marie-Soleil, APPARICIO, Philippe, THOUEZ, Jean-Pierre (2007) GISbased spatial analysis of child pedestrian accidents near primary schools in Montréal, Canada. Applied GIS, vol. 3, nº 4 , p. 1-18.

CLEVELAND, William S. et DEVLIN, Susan J. (1988) Locally weighted regression: An approach to regression analysis by local fitting. Journal of the American Statistical Association, vol.83, no403, p. 596- 610.

CLIFTON, Kelly J. et KREAMER-FULTS, Kandice (2007) An examination of the environmental attributes associated with pedestrian-vehicular crashes near public schools. Accident Analysis \& Prevention, vol. 39, n³4, p. 708-715.

DIEZ-ROUX, Anna Victoria (1998) Bringing context back into epidemiology: variables and fallacies in multilevel analysis. American Journal of Public Health, vol. 88, n 2 , p. 216-222.

DOUGHERTY, G., PLESS, B., WILKINS, R. (1990) Social class and the occurrence of traffic injuries and deaths in urban children. Canadian Journal of Public Health, vol. 81, n³, p. 204-209.

DUMBAUGH, Eric et RAE, Robert (2009) Safe urban form: Revisiting the relationship between community design and traffic Safety. Journal of the American Planning Association, vol.75, n³, p. 309-329.
ELVIK, Rune (2001) Area-wide urban traffic calming schemes: a meta-analysis of safety effects. Accident Analysis \& Prevention, vol. 33, nº 3, p. 327-336.

EWING, Reid et DUMBAUGH, Eric (2009) The built environment and traffic safety: A review of empirical evidence. Journal of Planning Literature, vol.23, no 4 , p. 347-367.

FEDERAL HIGHWAY ADMINISTRATION (2004) Signalized intersections: Informational guide. Report no. FHWAHRT-04-091. U.S. Department of Transportation.

GARDER, Per Erik (2004) The impact of speed and other variables on pedestrian safety in Maine. Accident Analysis \& Prevention, vol. 36, no 4 , p. 533-542.

GRUNDY, Chris, STEINBACH, Rebecca, EDWARDS, Phil, GREEN, Judith ARMSTRONG, Ben et WILKINSON, Paul (2009) Effect of 20 mph traffic speed zones on road injuries in London, 1986-2006: controlled interrupted time series analysis. British Medical Journal, n³39, p. b4469.

KINDIG, David et STODDART, Greg (2003). What is population health? American Journal of Public Health, vol. 93, n³, p. 380-383.

LAFLAMME, Lucie, BURROWS, Stéphanie, HASSELBERG, Marie (2009) Socioeconomic differences in injury risks - A review of findings and a discussion of potential countermeasures. World Health Organization.

LA SCALA, Elizabeth A., GRUENEWALD, Paul J. et JOHNSON, Fred W. (2004) An ecological study of the locations of schools and child pedestrian injury collisions. Accident Analysis \& Prevention, vol.36, $\mathrm{n}^{\circ} 4$, p. 569-576.

LEE, Chris et ABDEL-ATY, Mohamed (2005) Comprehensive analysis of vehicle-pedestrian crashes at intersections in Florida. Accident Analysis \& Prevention, vol.37, $\mathrm{n}^{\circ} 4$, p. 775-786. 
LICAJ, Idlir, HADDAK, Mouloud POCHET, Pascal et CHIRON, Mireille (2011) Contextual deprivation, daily travel and road traffic injuries among the young in the Rhône Département (France). Accident Analysis \& Prevention, vol.43, n5, p. 1617-1623.

LIGHTSTONE, A. S., DHILLON, P.K., PEEKASA, C. Et KRAUS, J.F. (2001) A geographic analysis of motor vehicle collisions with child pedestrians in Long Beach, California: comparing intersection and midblock incident locations. Injury Prevention, vol. 7 , no 2 , p. $155-160$.

LYON, Craig et PERSAUD, Bhagwant (2002) Pedestrian collision prediction models for urban intersections. Transportation Research Record 1818, nº 1, p. 102-107.

LYONS, R. A., JONES, S. J., NEWCOMBE, R. G. et PALMER S.R. (2006). The influence of local politicians on pedestrian safety. Injury Prevention, vol. 12, n5 5 , p. 312-315.

MORENCY, Patrick (2010) Potentiel d'une approche populationnelle orientée vers la reconfiguration des environnements urbains pour améliorer la sécurité des piétons. Université de Montréal. Département de médecine sociale et préventive, thèse de doctorat.

MORENCY, Patrick et CLOUTIER, Marie-Soleil (2006) From targeted "black spots" to areawide pedestrian safety. Injury Prevention, vol. 12, nº6, p. 360-364.

MIRANDA-MORENO, Luis F., MORENCY, Patrick et EL-GENEIDY, Ahmed M. (2010) The link between built environment, pedestrian activity and pedestrian-vehicle collision occurrence at signalized intersections. Accident Analysis \& Prevention, vol. 43, nº 5, p. 1624-1634.

NATIONAL HIGHWAY TRAFFIC SAFETY ADMINISTRATION (2008) National Pedestrian Crash Report - DOT HS 810968. U.S. Department of Transportation.

PEEK-ASA, Corinne et ZWERLING, Craig (2003) Role of environmental interventions in injury control and prevention. Epidemiologic Reviews, vol. 25, nº 1, p. 77-89.
PUBLIC HEALTH AGENCY OF CANADA (2005) Leading causes of death, Canada. [En ligne.] http://www.phac-aspc.gc.ca/ publicat/lcd-pcd97/table1-eng.php

PULUGURTHA, Srinivas S., KRISHNAKUMAR, Vanjeeswaran K. et NAMBISAN, Sashi S. (2007) New methods to identify and rank high pedestrian crash zones: An illustration. Accident Analysis \& Prevention, vol. 39, n 4 , p. 800-811.

RETTING, Richard A., FERGUSON, Susan A. et McCARTT, Anne T. (2003) A review of evidence-based traffic engineering measures designed to reduce pedestrianmotor vehicle crashes. American Journal of Public Health, vol. 93, n9 9, p. 1456-1463.

ROBERTS, Ian et CROMBIE, Iain (1995) Child pedestrian deaths: Sensitivity to traffic volume, evidence from the USA. Journal of Epidemiology and Community Health, nº49, p. 186-188.

ROBERTS, Ian, NORTON, R., JACKSON, R., DUNN, R. et HASSAL, I.(1995) Effect of environmental factors on risk of injury of child pedestrians by motor vehicles: a case-control study. British Medical Journal, vol. 310, p. 91-94.

RODGERS, S.E., JONES, S.J., MACEY, S.M., et LYONS, R.A. (2010) Using geographical information systems to assess the equitable distribution of traffic-calming measures: translational research. Injury Prevention, vol. 16, no 1 , p. 7-11.

ROSE, Geoffrey (1992) The strategy of preventive medicine. Oxford Medical Publications.

SCHOFIELD Grant M., GIANOTTI, Simon, BADLAND, Hannah et HINCKSON, Erica A. (2008) The incidence of injuries traveling to and from school by travel mode. Preventive Medicine, vol.46, p. 74-76.

SOCIÉTÉ DE L'ASSURANCE AUTOMOBILE DU QUEBEC (2005) Dossiers statistiques accidents, parc automobile, permis de conduire: bilan 2004. Québec. 
SOCIÉTÉ DE L'ASSURANCE AUTOMOBILE DU QUEBEC (2009) Dossiers statistiques accidents, parc automobile, permis de conduire: bilan 2008. Québec.

WIER, Megan, WEINTRAUB, June, HUMPHREYS, Elizabeth H., SETO, Edmund et BHATIA, Rajiv (2009) An area-level model of vehicle-pedestrian injury collisions with implications for land use and transportation planning. Accident Analysis \& Prevention, vol. 41, n 1, p. 137-145.

YIANNAKOULIAS, Niko, SMOYER-TOMIC, Karen E., HODGSON, John, SPADY, Donald, ROWE, Brian H. et VOAKLANDER, Donald C. (2002) The spatial and temporal dimensions of child pedestrian injury in Edmonton. Canadian Journal of Public Health, vol. 93, nº 6, p. 447-451. 